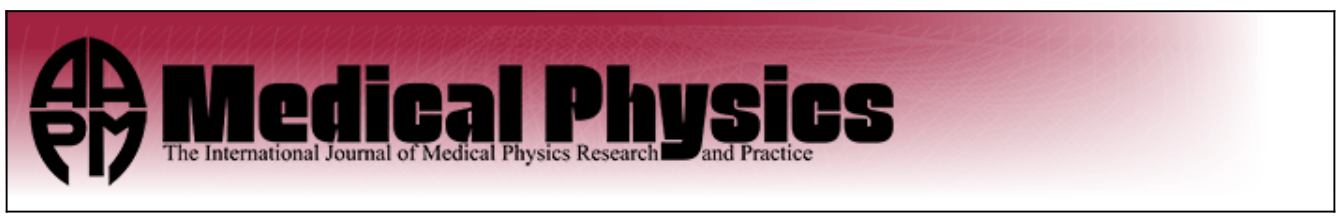

\title{
Dose ratio proton radiography using the proximal side of the Bragg peak
}

\section{P. J. Doolan, G. Royle, A. Gibson, H.-M. Lu, D. Prieels, and E. H. Bentefour}

Citation: Medical Physics 42, 1871 (2015); doi: 10.1118/1.4915492

View online: http://dx.doi.org/10.1118/1.4915492

View Table of Contents: http://scitation.aip.org/content/aapm/journal/medphys/42/4?ver=pdfcov

Published by the American Association of Physicists in Medicine

\section{Articles you may be interested in}

Camera selection for real-time in vivo radiation treatment verification systems using Cherenkov imaging

Med. Phys. 42, 994 (2015); 10.1118/1.4906249

Monitoring external beam radiotherapy using real-time beam visualization

Med. Phys. 42, 5 (2015); 10.1118/1.4901255

A real-time regional adaptive exposure method for saving dose-area product in $\mathrm{x}$-ray fluoroscopy Med. Phys. 40, 051911 (2013); 10.1118/1.4801908

Imaging of moving fiducial markers during radiotherapy using a fast, efficient active pixel sensor based EPID Med. Phys. 38, 6152 (2011); 10.1118/1.3651632

Proof of principle study of the use of a CMOS active pixel sensor for proton radiography Med. Phys. 38, 622 (2011); 10.1118/1.3496327
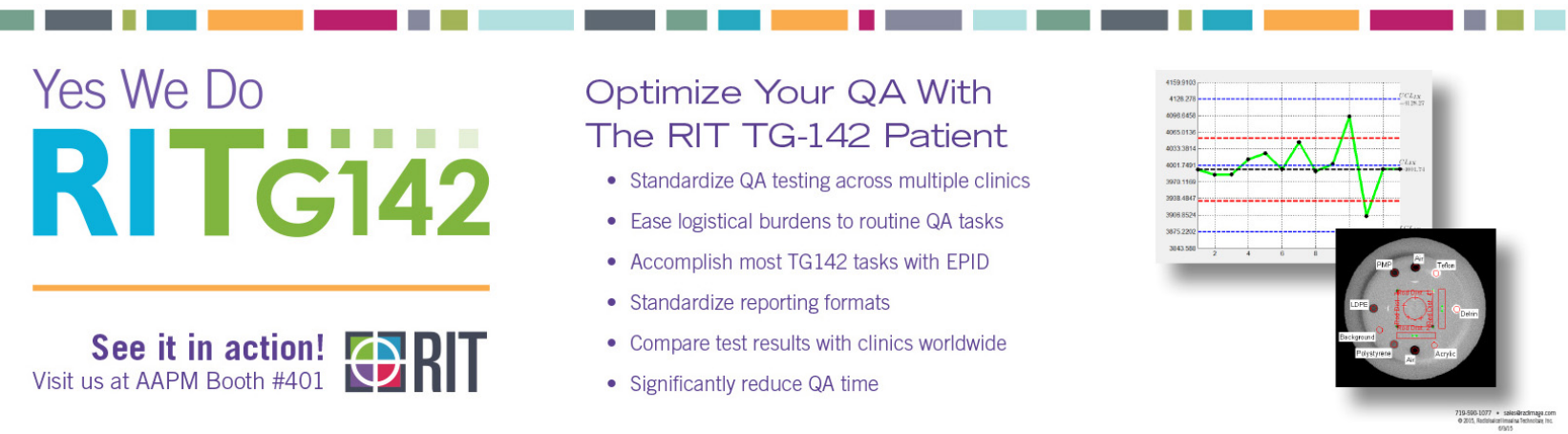


\title{
Dose ratio proton radiography using the proximal side of the Bragg peak
}

\author{
P. J. Doolan, ${ }^{\text {a) }}$ G. Royle, and A. Gibson \\ Department of Medical Physics and Bioengineering, University College London, \\ London WC1E 6BT, United Kingdom \\ H.-M. Lu \\ Department of Radiation Oncology, Massachusetts General Hospital, Boston, Massachusetts 02114 \\ D. Prieels and E. H. Bentefour \\ Ion Beam Applications (IBA), 3 Chemin du Cyclotron, Louvain la Neuve B-1348, Belgium
}

(Received 25 June 2014; revised 14 January 2015; accepted for publication 8 March 2015; published 25 March 2015)

Purpose: In recent years, there has been a movement toward single-detector proton radiography, due to its potential ease of implementation within the clinical environment. One such single-detector technique is the dose ratio method in which the dose maps from two pristine Bragg peaks are recorded beyond the patient. To date, this has only been investigated on the distal side of the lower energy Bragg peak, due to the sharp falloff. The authors investigate the limits and applicability of the dose ratio method on the proximal side of the lower energy Bragg peak, which has the potential to allow a much wider range of water-equivalent thicknesses (WET) to be imaged. Comparisons are made with the use of the distal side of the Bragg peak.

Methods: Using the analytical approximation for the Bragg peak, the authors generated theoretical dose ratio curves for a range of energy pairs, and then determined how an uncertainty in the dose ratio would translate to a spread in the WET estimate. By defining this spread as the accuracy one could achieve in the WET estimate, the authors were able to generate lookup graphs of the range on the proximal side of the Bragg peak that one could reliably use. These were dependent on the energy pair, noise level in the dose ratio image and the required accuracy in the WET. Using these lookup graphs, the authors investigated the applicability of the technique for a range of patient treatment sites. The authors validated the theoretical approach with experimental measurements using a complementary metal oxide semiconductor active pixel sensor (CMOS APS), by imaging a small sapphire sphere in a high energy proton beam.

Results: Provided the noise level in the dose ratio image was $1 \%$ or less, a larger spread of WETs could be imaged using the proximal side of the Bragg peak (max $5.31 \mathrm{~cm}$ ) compared to the distal side ( $\max 2.42 \mathrm{~cm}$ ). In simulation, it was found that, for a pediatric brain, it is possible to use the technique to image a region with a square field equivalent size of $7.6 \mathrm{~cm}^{2}$, for a required accuracy in the WET of $3 \mathrm{~mm}$ and a $1 \%$ noise level in the dose ratio image. The technique showed limited applicability for other patient sites. The CMOS APS demonstrated a good accuracy, with a root-mean-square-error of $1.6 \mathrm{~mm}$ WET. The noise in the measured images was found to be $\sigma=1.2 \%$ (standard deviation) and theoretical predictions with a $1.96 \sigma$ noise level showed good agreement with the measured errors.

Conclusions: After validating the theoretical approach with measurements, the authors have shown that the use of the proximal side of the Bragg peak when performing dose ratio imaging is feasible, and allows for a wider dynamic range than when using the distal side. The dynamic range available increases as the demand on the accuracy of the WET decreases. The technique can only be applied to clinical sites with small maximum WETs such as for pediatric brains. () 2015 Author(s). All article content, except where otherwise noted, is licensed under a Creative Commons Attribution 3.0 Unported License. [http://dx.doi.org/10.1118/1.4915492]

Key words: single-detector proton radiography, proton therapy, WEPL, WET, dose ratio method

\section{INTRODUCTION}

Proton radiography, first proposed in the $1960 \mathrm{~s},{ }^{1}$ is based on measuring the position and energy loss of protons after passing through a medium. This information is then utilized to estimate the integral energy loss along the trajectory, which is converted into a material water-equivalent thickness (WET).

Proton radiography offers the potential for higher tissue-totissue contrast and better density resolution than $\mathrm{x}$-ray imaging because of the sharpness of the Bragg peak near the end of the proton range. ${ }^{2,3}$ The shape of the proton depth dose curve enables the use of less dose. ${ }^{2,4}$ One of the major advantages of proton radiography is the potential to generate relative stopping power (RSP) maps of the patient directly, rather than the current process of converting the patient's X-ray CT dataset using a calibration curve. ${ }^{5}$ This process is thought to contribute between $0.5 \%$ and $1.8 \%$ to the proton beam range uncertainty, depending on whether the uncertainties in the mean material 
ionization energies are considered. ${ }^{6,7}$ Although reconstruction of multiple proton radiographic projections (known as "proton CT") is required to generate a 3D map of the patient RSP, the acquisition of proton radiographs in a single direction can still prove useful. Previously, it has been shown that the patientspecific calibration curves (converting the x-ray CT dataset to units of RSP) can be generated through an optimization procedure together with the original $\mathrm{x}$-ray $\mathrm{CT} .{ }^{8}$ In addition to reducing the proton beam range uncertainty in proton treatment planning, proton radiography has a number of other potential applications, including convenient patient setup, ${ }^{9}$ passive range verification, ${ }^{8}$ and active range verification. ${ }^{10}$

One of the major disadvantages of proton radiography is due to the fact that protons undergo multiple Coulomb scattering (MCS) in the patient, which limits the spatial resolution compared to $\mathrm{x}$-ray imaging. ${ }^{11}$ This physical process has driven the classical approach to proton radiography in which attempts are made to correct for MCS by tracking protons before and after the patient and by measuring the residual proton energy at the patient exit. The proton trajectory and the energy loss across that path can then be estimated. To keep the patient dose to a minimum, this technique requires the tracking of every proton, which places high demands on the speed of device (typical proton dose rates are on the order of $10^{9}$ protons/s). A range of designs have been used for this high speed proton tracking: scintillators, ${ }^{4,12,13}$ semiconductors ${ }^{14-17}$ plasma panels, ${ }^{18,19}$ nuclear emulsion films, ${ }^{20}$ and gas-based devices. ${ }^{21,22}$ The energy measurement is either performed directly, using a calorimeter, or inferred from the proton range, in a range telescope design. A variety of direct methods have been investigated: magnetic spectrometry, ${ }^{23} \mathrm{NaI}$ calorimeters ${ }^{24} \mathrm{CsI}$ calorimeters, ${ }^{15,25,26}$ and scintillating crys- tals. ${ }^{13,27}$ Range telescope designs have included stacks of scintillators $^{21,28}$ and multilayer ionization chambers. ${ }^{29,30}$

Despite the promises and the extent of research on the topic, proton radiography has not been routinely introduced into the clinical environment. The classical devices are generally complex and they can often be bulky and the projected cost is high. As such, there has been a recent movement toward using only a single detector positioned beyond the patient. A variety of technologies have been explored, including fluorescent screens coupled to CCD cameras, ${ }^{31-33}$ commercial flat-panel detectors, ${ }^{34}$ and complementary metal oxide semiconductor active pixel sensors (CMOS APS). 3,17,35

The measurement of dose offers a simple approach to proton radiography. A range of such techniques have been tested, including using pairs of sloped spread-out Bragg peaks (SOBPs), ${ }^{36,37}$ measuring the time-resolved dose from a proton beam passively scattered by a range modulator wheel, ${ }^{10,38,39}$ and by measuring the ratio of doses of two pristine Bragg peaks ${ }^{40}$ which is the subject of this work. The technique works as follows: (1) measure depth dose curves in water for two pristine Bragg peaks; (2) determine the "dose ratio curve" for the energy pair by dividing the lower energy by the higher energy; (3) record the dose map beyond an object of unknown thickness/material for each of the two energies and calculate the dose ratio map; and (4) convert to a WET using the dose ratio curve. This approach, known as the dose ratio method, is shown schematically in Fig. 1.

This method has a number of advantages. It requires the use of a single imaging detector, which is more convenient and more likely to fit into the clinical environment than the conventional trackers and energy measurement setup. Also, by taking the dose ratio, the detector does not need to be (a)

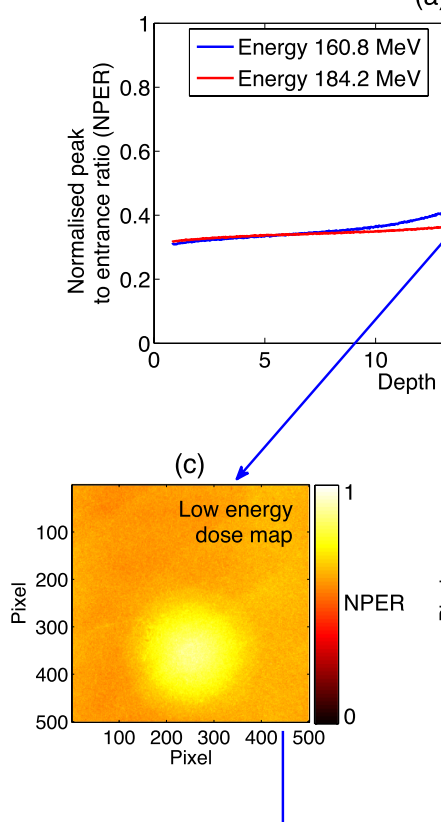

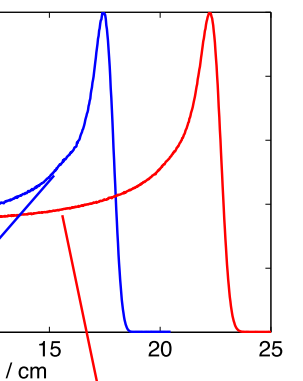
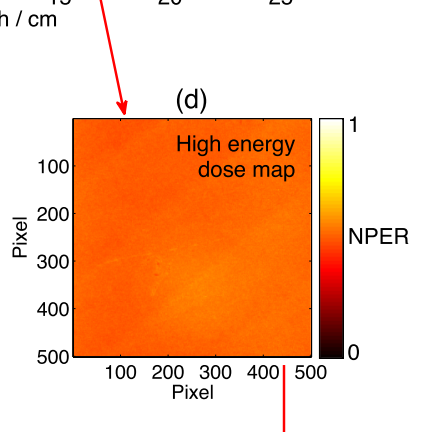

(b)
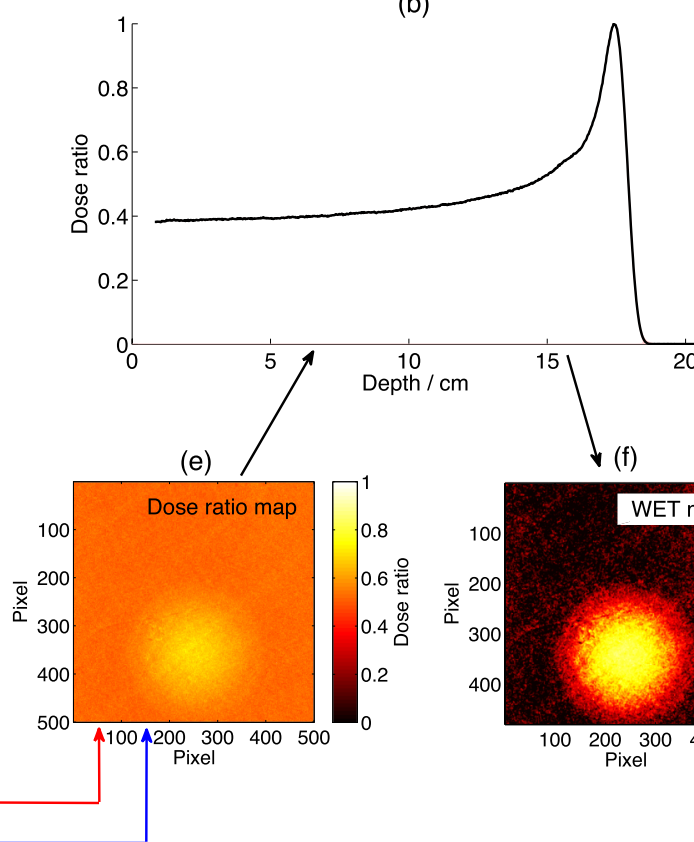

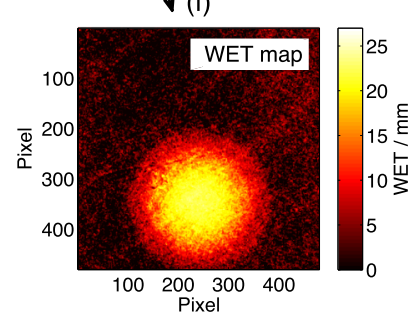

FIG. 1. Dose ratio method steps to produce the dose ratio image. (a) Measure depth dose curves in water for two pristine Bragg peaks. (b) Determine the dose ratio curve. (c) and (d) Record the dose map beyond an object for each of the two energies. (e) Form a dose ratio map by dividing (c) by (d). Finally, convert the dose ratio map to a WET map (f) using the dose ratio curve. 
(a)

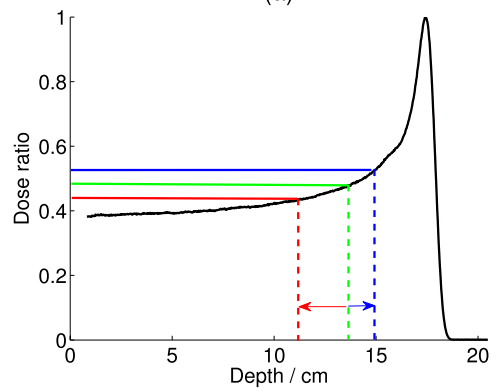

(c)

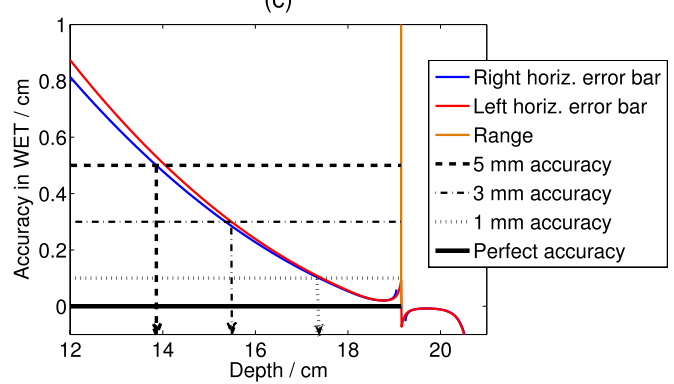

(b)

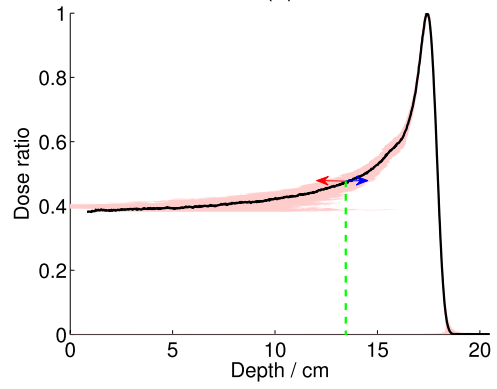

(d)

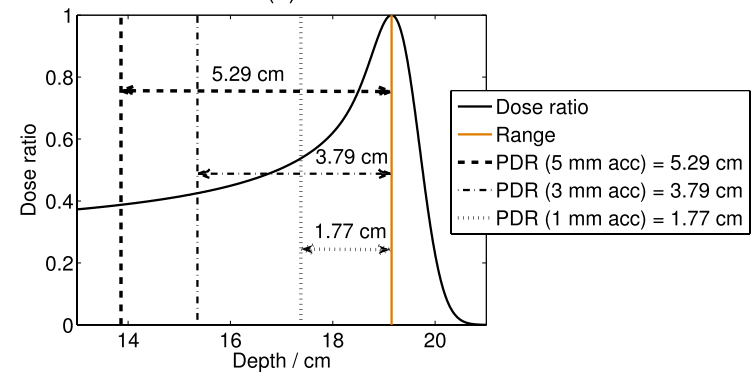

FIG. 2. Method of determining the proximal dynamic range. (a) Uncertainties in the dose ratio translate into uncertainties in the WET (depth). (b) These are added to the dose ratio curve as horizontal error bars. (c) The error bar magnitude defines the accuracy in the WET. (d) Dependent on the required accuracy, the proximal dynamic range can be determined.

able to measure absolute dose, rather it must demonstrate high reproducibility. The technique also has the potential to be applied to pencil beam scanning (PBS), as it uses pristine Bragg peaks rather than the spread-out Bragg peaks used in passive scattering.

It has been previously shown that this technique can achieve millimeter accuracy in determining the WET, using the distal edge of the lower energy Bragg peak. ${ }^{40}$ However, in this approach, the dynamic range is limited by the steep falloff and the approximate material WET must be known to a high accuracy to appropriately select the energy pair. Although on the proximal side of the Bragg peak, the falloff is only from $100 \%$ to $\sim 30 \%$ (compared to the distal falloff from $100 \%$ to $0 \%$ ), the falloff is much less steep, leading to a potentially much larger dynamic range. It has not yet been explored how much of the proximal side of the Bragg peak can be reliably used and for which patient treatment sites this imaging technique could be applied. In this paper, we aim to do the following.

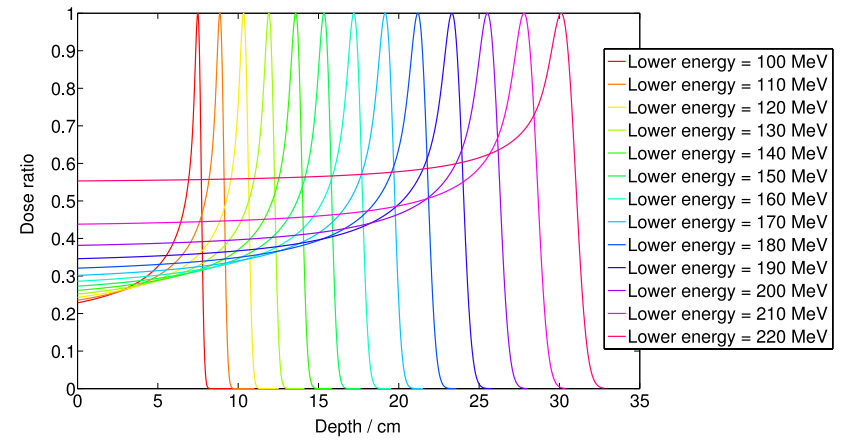

FIG. 3. Normalized theoretical dose ratio curves (all paired with $230 \mathrm{MeV}$ ). (i) Investigate the theoretical limits of the dose ratio method for the proximal side of the Bragg peak. We determine the dynamic range for different energy pairs, different required accuracies in the WET, and for a range of uncertainties in the dose ratio map.

(ii) Determine the applicability of this technique. We follow the same theoretical analysis as in (i), for the energy pair most appropriate to the particular clinical site, and then determine the area that can be imaged under different sets of clinical requirements and proton imager performances.

(iii) Validate the theoretical predictions with real measurements using a CMOS APS. After measuring the noise in the CMOS APS, we generate theoretical limits and verify that the accuracy in imaging a sapphire sphere matches these predictions.

\section{METHODS AND MATERIALS}

\section{A. Theoretical limits}

The dose ratio method relies on the measurement of pristine Bragg peaks in water. To assess the theoretical limits of the technique, independent on the detector used, we modeled pristine Bragg peaks of different energies using the analytical approximation that considers both range straggling and an initial spread of beam energies. ${ }^{41}$ The range $R_{0}$ was approximated by the range-energy relation $R_{0}=\alpha E^{p}$, for a proton beam with initial energy $E$, where the constants $\alpha=0.0022$ and $p=1.77$ are for a proton beam in water. ${ }^{41}$ The width of the Gaussian energy spectrum was set to $\sigma_{E}=0.01 E$. Taking the ratio of normalized depth doses curves of two energies, a theoretical dose ratio curve can be generated. 
Our approach to determining the theoretical limits, shown schematically in Fig. 2, is based on introducing uncertainties in dose ratio measurement and inspecting the change in the WET estimate from the theoretical dose ratio curve [in Fig. 2(a), the original dose ratio value is shown in the center with upper/lower uncertainties above/below]. Then, right/left horizontal error bars are added to the dose ratio curve for that particular dose ratio value [shown as right/left arrows in Fig. 2(b)]. For a given introduced uncertainty, we used the spread in the proton WET values to define the maximum achievable accuracy of the WET measurement [Figs. 2(c) and 2(d)]. The higher the accuracy in the WET that is required by the user, which is dependent on the size of the horizontal error bars, the smaller the amount of the proximal side of the Bragg peak (henceforth referred to as "proximal dynamic range") that can be used for imaging.

In this analysis, the impact of an uncertainty in the dose ratio map of $1 \%$ to $10 \%$ (in steps of $1 \%$ ) is investigated. Uncertainties in the individual energy images will combine in quadrature in the dose ratio map, with typical values of standard deviation $(\sigma)$ in the dose ratio map found to be $1 \%-3 \%$. In real measurements, uncertainties will arise in the raw measured images from both statistical uncertainties (dependent on the number of protons) and detector uncertainties (such as quantum noise, shot noise, read noise, and fixed pattern noise). To remove the effect of statistical uncertainties, which would otherwise vary across the Bragg peak, the error in the dose ratio was divided by the normalized dose at each depth.

It is desirable to set the higher energy to always be the highest deliverable by the machine, so that a flat proximal dose contributes to the dose ratio. Most proton machines have a maximum deliverable energy of $230 \mathrm{MeV}$, so in this analysis, dose ratio curves were formed using pairs where the maximum energy was fixed at $230 \mathrm{MeV}$. The lower energy varied between 100 and $220 \mathrm{MeV}$, in steps of $10 \mathrm{MeV}$, producing 13 theoretical dose ratio curves, as shown in Fig. 3. For all these settings, proximal dynamic ranges were calculated for required accuracies of 1 to $5 \mathrm{~mm}$. For comparison, the analysis was also repeated on the distal edge of the Bragg peak and distal dynamic ranges were calculated.

Most proton centers currently add a margin of between $2.5 \%$ and $3.5 \%$ of the proton range to account for range uncertainty, ${ }^{7}$ so a radiography technique that could predict the range to a higher accuracy than this would be an improvement on current practice. For a typical therapeutic proton beam with a range of $20 \mathrm{~cm}$, accuracies we are investigating $(1-5 \mathrm{~mm})$ correspond to range uncertainties of $0.5 \%-2.5 \%$, which would offer an improvement in current proton beam range predictions.

\section{B. Application to patient sites}

The results of the theoretical analysis (of Sec. 2.A) are two curves for each energy pair in which the proximal dynamic range is calculated for either a given required WET accuracy or for a given percentage error in the dose ratio. Using these curves, it is possible to illustrate the application of the technique for different patient sites.
For any patient site, there will be a range of WETs. Due to the shape of the proton depth dose curve (Fig. 3), the energy pair must be carefully selected so that the lower energy exits the patient and an image can be recorded. This lower energy must, therefore, have a range higher than that of the thickest WET in the patient site. Once the appropriate energy pair has been selected, the proximal dynamic range can be retrieved from the analysis of Sec. 2.A, for different required accuracies in the WET and for different noise levels in the dose ratio image. The steps for determining the region that can be imaged is as follows.

1. Convert the x-ray CT dataset of each patient to RSPs using a calibration curve. For this, we used our departmental tissue-substitute calibration curve, which is formed of two straight lines (one with a gradient of 0.001 between $\mathrm{HU}=-1000$ and 40 , and one with a gradient of 0.0005 from $\mathrm{HU}=40$ to 2995) and a correction above $\mathrm{HU}=2996$ to account for titanium. It is then necessary to perform parallel line ray tracing through the volume [we conducted this using the open-source software, PLASTIMATCH (plastimatch.org)].

2. Compute the WET digitally reconstructed radiograph (DRR) by summing up the RSPs along the rays through the patient.

3. Calculate the maximum WET through the patient and convert to a minimum energy to the distal edge using the range-energy relation (we rounded these up to the nearest $10 \mathrm{MeV}$ ).

4. Using the theoretical results for the specific energy pair (analysis of Sec. 2.A), determine the WETs that lie within the proximal dynamic range.

All DRRs were produced with parallel ray tracing and with a pixel size of $1 \mathrm{~mm}$, as shown in Fig. 4. The cropped field sizes are prostate, $61 \times 101 \mathrm{~mm}=6161 \mathrm{~mm}^{2}$; lung, solid cropped field, $41 \times 46 \mathrm{~mm}=1886 \mathrm{~mm}^{2}$; and lung, dashed cropped field, $34 \times 35 \mathrm{~mm}=1190 \mathrm{~mm}^{2}$. Details of the maximum WET in the image, the corresponding range, and the lower energy selected are provided in Table I.

\section{C. Experimental demonstration in a single-detector CMOS APS}

The purpose of the experimental measurements was twofold: (1) to demonstrate that it is possible to acquire accurate images using the dose ratio proton radiographic method with a single CMOS APS and (2) to show that the uncertainties in these measurements follow the theoretical predictions.

\section{C.1. Experimental details}

Measurements were made at the Francis H. Burr Proton Therapy Facility, Massachusetts General Hospital. To ensure a suitably large spot size was used, covering the whole sensor, the beam was used in single scattering mode. Depth dose curves for the two energies, with ranges $(R 90)$ of 17.7 and $22.5 \mathrm{~cm}$, were acquired using a Markus chamber on a stepper 
(a)

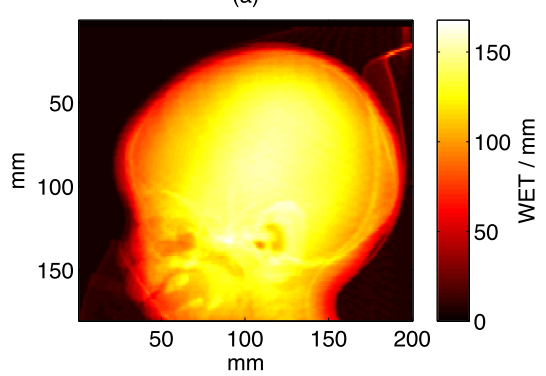

(d)

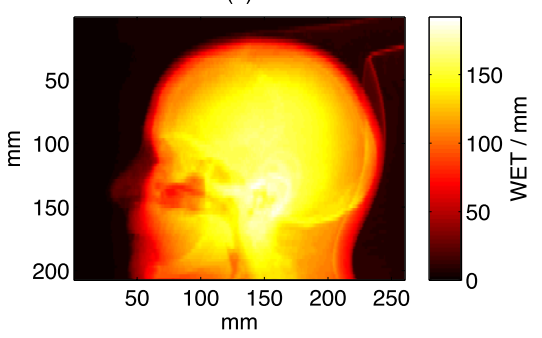

(g)

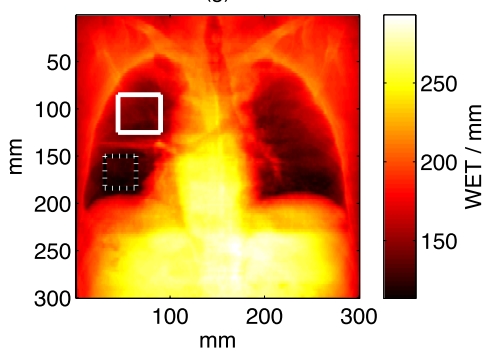

(b)

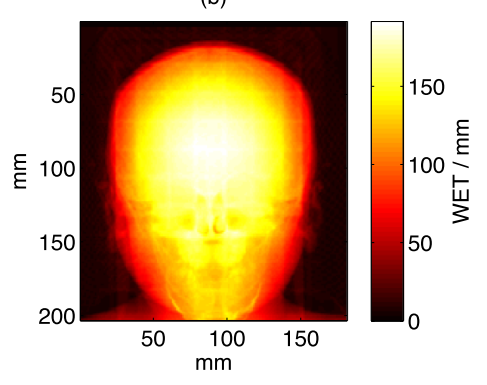

(e)

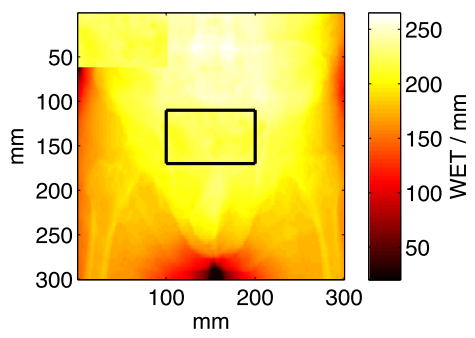

(h)

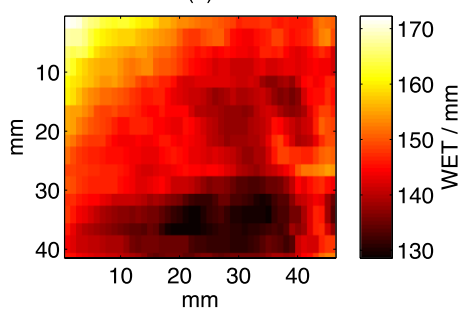

(c)

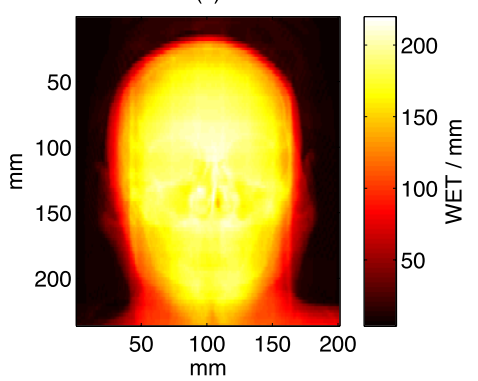

(f)

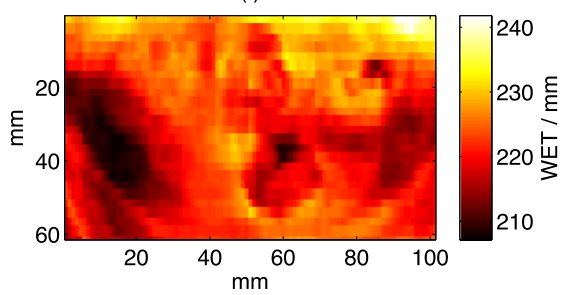

(i)

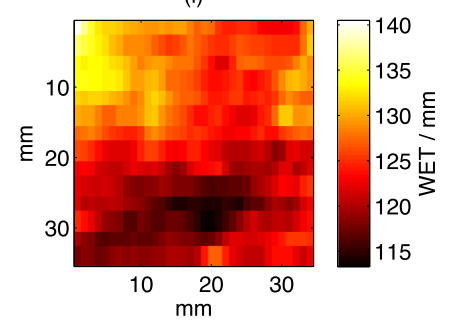

FIG. 4. DRRs of the different patient sites to be analyzed: pediatric brain in (a) LR and (b) AP directions; adult brain in (c) AP and (d) LR directions; (e) full prostate field-of-view and (f) cropped region to analyze; (g) full lung field-of-view, with (h) AP1 (solid) and (i) AP2 (dashed) cropped regions of interest (ROIs).

motor in a water tank, together with a reference entrance chamber. This arrangement is shown in Fig. 5.

Dose ratio measurements were made using a CMOS APS, "Vanilla." CMOS APSs have shown promise in imaging for proton radiography, ${ }^{3,17}$ with advantages such as low manufacturing costs, efficient charge collection, high speed imaging, high spatial resolution, and ROI addressability. Full details of Vanilla and an assessment of its performance can be found in the literature, ${ }^{42}$ but for our application, the important features are a sensitive area of $13 \times 13 \mathrm{~mm}$, comprised of an array of $520 \times 520$ pixels each with $25 \mu \mathrm{m}$ pitch and repetition rates between 1 and 1000 frames per second (fps) (dependent on the ROI addressed). In our measurements, we imaged the full sensitive area at a frame rate of $44 \mathrm{fps}$. Dose optimization was not part of the scope of the project and in forming the dose ratio maps, approximately $0.8 \mathrm{mGy}$ was delivered per frame.

\section{C.2. Dose ratio measurements with a sapphire sphere}

Due to our small sensor size, we imaged a small red sapphire $\left(\mathrm{Al}_{2} \mathrm{O}_{3}\right.$, doped with Chromium, $\left.\mathrm{Cr}_{2} \mathrm{O}_{3}\right)$ sphere, with diameter $6.35 \mathrm{~mm}$. As detailed in Secs. 2.A and 2.B, there is a limited dynamic range using the dose ratio radiographic method, so to

TABLE I. Clinical sites investigated for applicability of dose ratio proton radiography.

\begin{tabular}{lcccc}
\hline \hline Case & Field & $\begin{array}{c}\text { Maximum } \\
\text { WET/cm }\end{array}$ & $\begin{array}{c}\text { Minimum energy to } \\
\text { distal edge/MeV }\end{array}$ & $\begin{array}{c}\text { Energy rounded up to } \\
\text { the nearest 10 MeV }\end{array}$ \\
\hline Pediatric brain & AP & 19.2 & 168.2 & 170 \\
Adult brain & LR & 16.8 & 156.1 & 160 \\
Prostate & AP & 22.0 & 181.9 & 190 \\
Lung & LR & 19.2 & 168.5 & 170 \\
& AP & 24.2 & 191.9 & 200 \\
\hline \hline
\end{tabular}

Note: AP, anterior-posterior; LR, left-right; the cropped fields for the lung, AP1 and AP2, are the solid and dashed ROIs for the lung patient. 


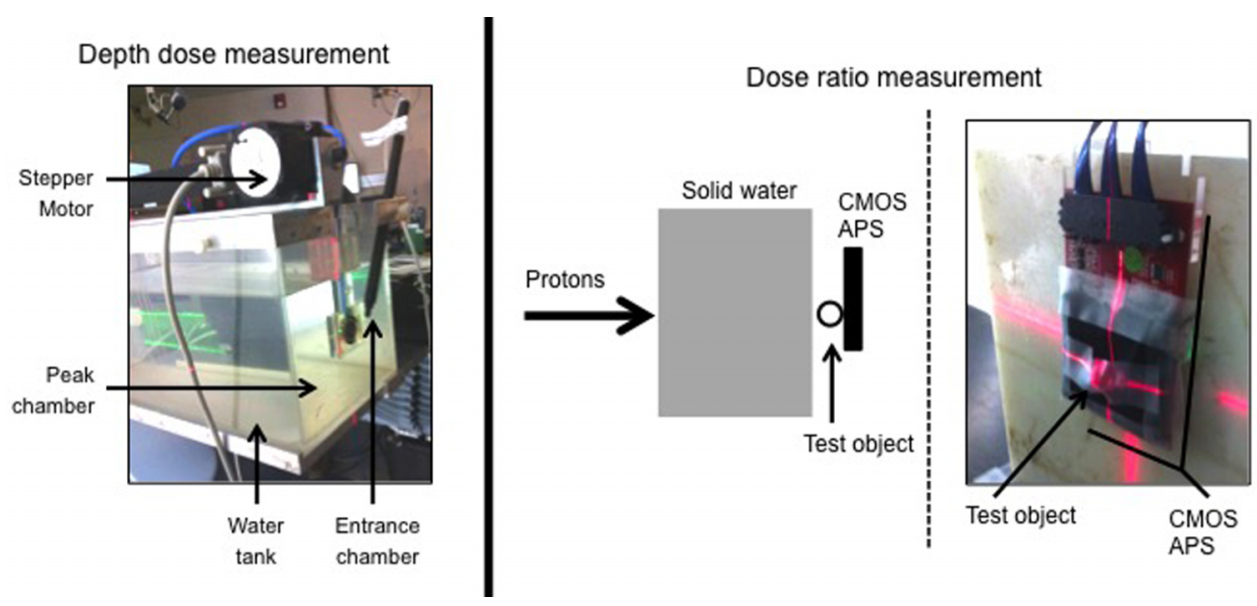

Fig. 5. (Left) Experimental setup for depth dose measurements. (Right) Schematic and photograph of experimental setup for dose ratio measurements.

ensure we used the sensitive part of the Bragg peak, we needed to place solid water slabs between the object and Vanilla, as shown in Fig. 5. The level of $\mathrm{Cr}_{2} \mathrm{O}_{3}$ doping was unknown for our sample so a percentage weight of $0.05 \%$ was assumed, in accordance with typical literature values for such synthetically grown crystals. ${ }^{43,44}$ For such a composition, the effective atomic number $Z_{\text {eff }}$ was calculated to be 11.40 (Ref. 45) and the relative electron density $\rho_{e}$ was calculated to be $3.51 .{ }^{46}$ The RSP was estimated using the empirical formula in Eq. (1), ${ }^{47}$

$$
\mathrm{RSP}=\rho_{e}\left(12.77-\left(0.098^{*} Z_{\mathrm{eff}}+3.376\right)\right) / 8.45
$$

and found to be 3.44 , giving a maximum sphere WET of $21.8 \mathrm{~mm}$ (sphere diameter, $6.35 \mathrm{~mm}$, multiplied by RSP, 3.44). As we wanted to demonstrate the use of the proximal side of the Bragg peak only, solid water was added before the phantom with a thickness of $14.27 \mathrm{~cm}$ WET, such that the total thickness was less than the range of the lower energy beam $(17.7 \mathrm{~cm})$.

\section{C.3. Detector sensitivity calibration}

The CMOS sensor is an optical sensor, not designed for the direct detection of charged particles, and as such, it is not radiation hard. Decreases in detector sensitivity have been observed with increasing dose in other CMOS sensors, ${ }^{48-50}$ and the effect was also evident in our detector. The grayscale of the output image of Vanilla is usually distributed over 4096 digital numbers, but this dynamic range decreases as the sensitivity decreases. As our detector could not be triggered to acquire when the beam switches on, however, the dose to the detector was not carefully controlled and it was impossible to apply a sensitivity correction. To resolve this problem, the detector was calibrated before each acquisition, using a minimal dose (ten frames only) through a known material thickness.

\section{C.4. Image processing}

After averaging over 190 frames, images were processed in the same manner as the dose ratio curve was produced: (1) normalize the grayscale images for each energy and (2) divide the lower energy by the higher energy to form a dose ratio image. Before converting the dose ratio image to a WET map, we applied two corrections. The first correction was to remove the fixed pattern noise, induced by the manner of the readout of the CMOS sensor. If the flux had been identical for the two energies, the fixed pattern noise would have been identical $^{51}$ and the ratio of the images would have led to it canceling out. As this was not the case, we needed to use an interpolation notch-reject filter to the affected region on the Fourier transform of the image, ${ }^{52}$ the process of which is shown in Fig. 6. Second, a $3 \times 3$ median filter was passed over the image to remove the salt and pepper noise and the outer 20 pixels (in every direction) were cropped to remove discrepancies at the detector edge. The dose ratio image was then converted to WET using the measured dose ratio curve. (a)

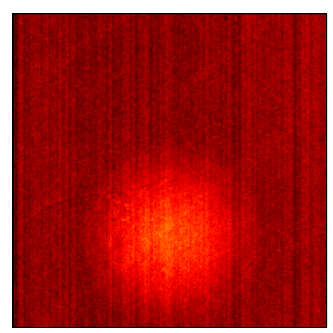

(b)

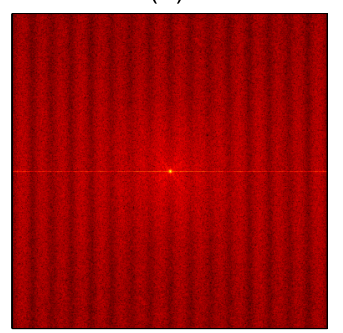

(c)

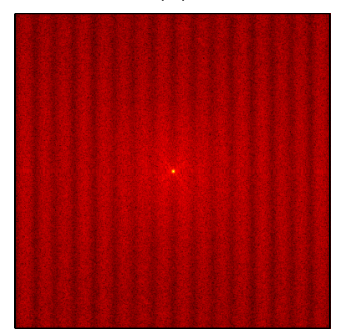

(d)

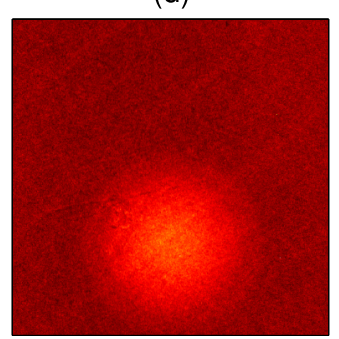

FIG. 6. Fixed pattern noise removal. (a) Raw dose ratio image. The vertical stripes are caused by fixed pattern noise. (b) Fourier transform of the dose ratio image, before correction, and (c) after applying the interpolation notch-reject filter. (d) Corrected, dose ratio image. 


\section{RESULTS}

\section{A. Theoretical limits}

After following the steps in Sec. 2.B, the user has the correct energy pair for the site to be imaged. An example dose ratio curve (with a lower energy of $170 \mathrm{MeV}$ ), with right and left error bars for $1 \%$ and $3 \%$ uncertainties in the dose ratio, is shown in Fig. 7. Plotting these error bars across the Bragg peak (also shown in Fig. 7) allows for calculation of the proximal dynamic range.

The proximal dynamic range is a function of the lower energy $(E 1)$ selected, the noise in the dose ratio map, and the required accuracy in the WET. The theoretical relationship between all of these factors is shown in Fig. 8, for a selection of noise levels. It should be noted that, for some energy pairs, if the noise level is too high and the required accuracy in the WET is too strict, no part of the Bragg peak can be used for imaging [thus, the lines in Fig. 8(a) are shortened]. For comparison, the analysis was repeated on the distal side of the Bragg peak and distal dynamic ranges were calculated, which are also shown in Fig. 8 with dashed lines. Unlike the proximal side, the distal dynamic ranges are almost independent of the required accuracy in the WET.

\section{B. Application to patient sites}

The theoretically maximum size of the area that can be imaged for each of the clinical sites is detailed in Table II. For reasons of brevity, only noise levels between $1 \%$ and $3 \%$ and for WET accuracies of 1-3 $\mathrm{mm}$ are included as these are the most clinically relevant. For the lung and prostate cases, the region being analyzed is already a cropped version of the full DRR, so results are displayed in terms of percentages (of the cropped region size). Areas greater than an equivalent $50 \times 50 \mathrm{~mm}^{2}$ field, or covering more than $75 \%$ of the cropped region, are shown in bold and underlined typefaces.

As an example, the areas that can be imaged for the AP pediatric brain are shown in Fig. 9 as black regions superimposed on the original WET DRR, for different noise levels in the dose ratio and for different required accuracies in the WET. Similar analyses were conducted for all the patient sites tested.

\section{C. Experimental demonstration in a single-detector CMOS APS}

\section{C.1. Accuracy and suitability of CMOS APS}

The measured dose ratio curve is shown in Fig. 10(a). The theoretical and measured WET maps are shown in Figs. 10(b) and $10(\mathrm{c})$, respectively. The difference between the theoretical and measured WET maps for the sapphire sphere is shown in Fig. 10(d). The location of the theoretical center of the sphere was determined by moving its center until the root-meansquare-error (RMSE) of the difference within the sphere radius [Fig. 10(e)] was minimal. The $\mathrm{max} / \mathrm{min} /$ mean errors were found to be $1.23 /-1.10 /-0.05 \mathrm{~cm}$ within the sphere radius. The RMSE and standard deviation were found to be 0.16 and $0.20 \mathrm{~cm}$, respectively. In Fig. 10(f), the measured WET values as a function of distance from the center of the sphere were determined (after rebinning the image into $0.1 \mathrm{~mm}$ pixels).

\section{C.2. Validation of theoretical predictions}

The calibration dose ratio image (acquired using a single solid water thickness, with no sphere), after rebinning into (a)

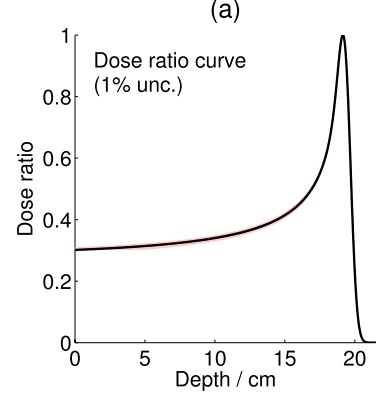

(d)

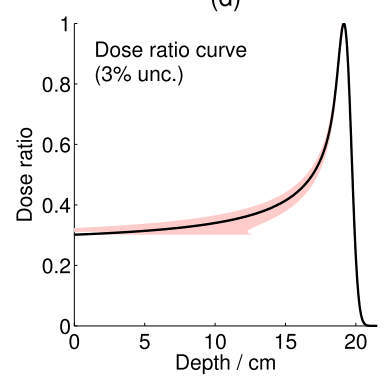

(b)

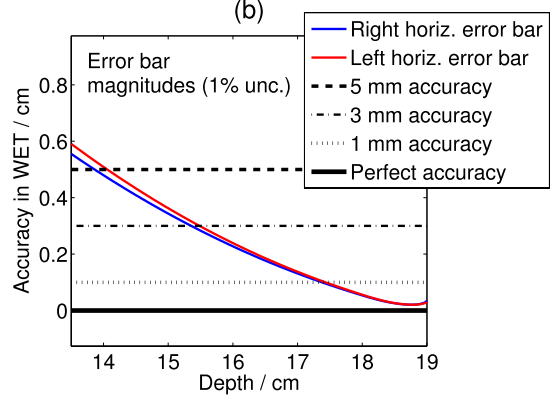

(e)

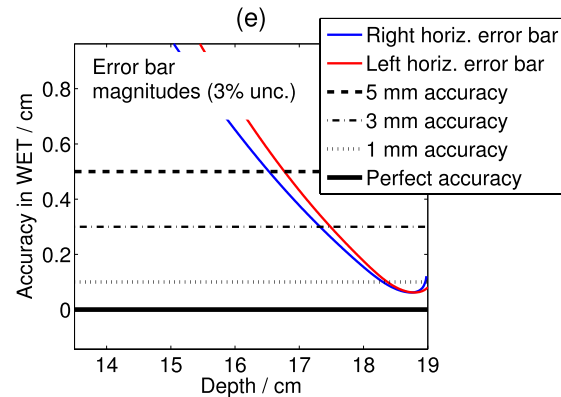

(c)
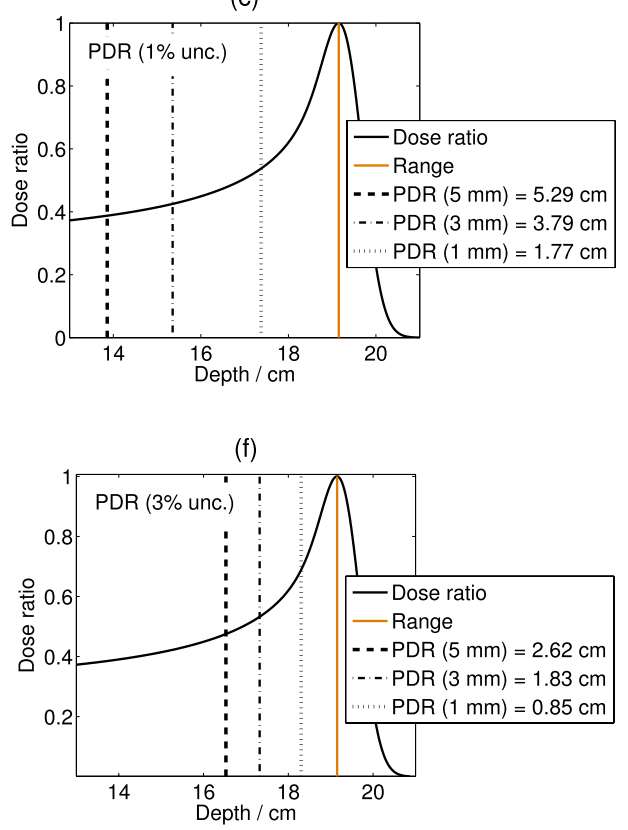

FIG. 7. (a) Dose ratio curve for the $170 / 230 \mathrm{MeV}$ energy pair, with horizontal error bars indicating the change in WET for a $1 \%$ uncertainty (unc.) in the dose ratio. (b) Magnitude of error bars plotted as a function of the depth, with different accuracies indicated. (c) Proximal dynamic ranges, for different required accuracies, shown with respect to the original dose ratio curve. (d)-(f) Same as (a)-(c), but for a 3\% uncertainty in the dose ratio. 
(a)

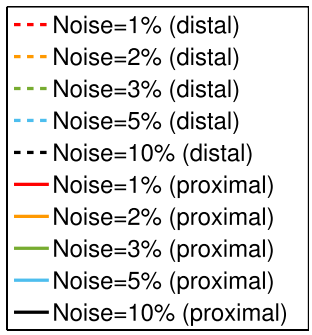

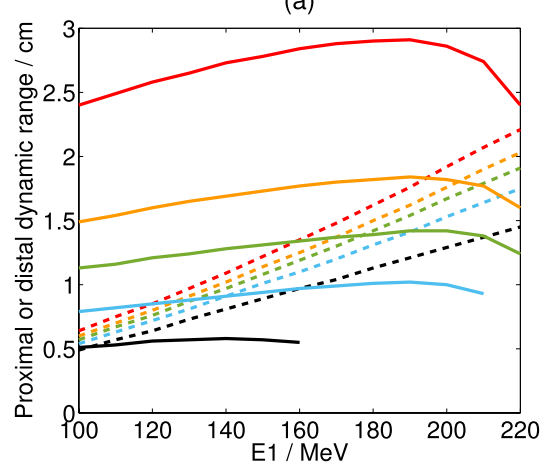

(b)

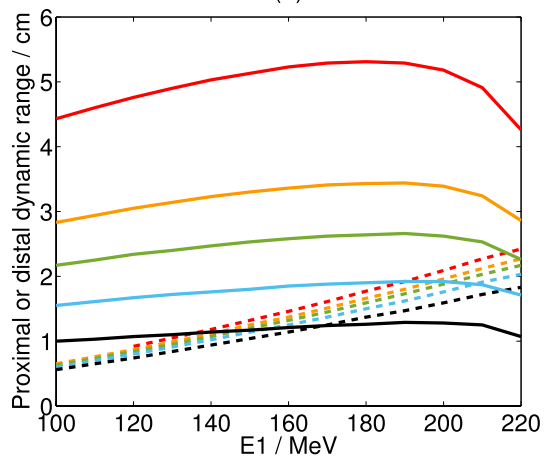

FIG. 8. Comparison of proximal (solid lines) and distal (dashed lines) dynamic ranges for required accuracies in the WET of (a) $2 \mathrm{~mm}$ and (b) $5 \mathrm{~mm}$.

$0.1 \mathrm{~mm}$ pixels, was found to have a standard deviation, $\sigma$ $=1.2 \%$. It can be seen in Fig. 10(e) that the error in the WET follows an approximately Gaussian shape. If we set the uncertainty in the dose ratio to be $1.96 \sigma$, or $2.35 \%$, we would expect $95 \%$ of our results to fall below the error bars of our theoretical predictions. To test this, we determined the theoretical limits using the same procedure as in Sec. 2.A, for the two energies used in the measurements (160.8 and 184.2 $\mathrm{MeV})$. The theoretical error bars for a change of the dose ratio of $2.35 \%$, together with the absolute measured WET errors, are shown in Fig. 11. The experimental measurements fall below the theoretical right/left horizontal error bars in $89.1 \% / 93.9 \%$ of cases, respectively.

\section{DISCUSSION}

\section{A. Theoretical limits}

Using the analytical approximation for the Bragg peak, we were able to define theoretical limits for the dose ratio method on the proximal side of the Bragg peak. Horizontal right and left error bars were added to theoretical dose ratio curves and used to determine the proximal dynamic range for varying energy pairs and different levels of noise in the dose ratio map. A requirement from the user is the knowledge of the expected uncertainty in their dose ratio map (defined in our study to be dose independent), which is a quadratic sum of the uncertainties in the images from each energy. For a

TABLE II. Magnitude of areas, for a variety of clinical sites, that can be imaged using dose ratio proton radiography.

\begin{tabular}{|c|c|c|c|c|c|}
\hline Case & Field & $\begin{array}{l}\text { Accuracy in } \\
\mathrm{WET} / \mathrm{mm}\end{array}$ & $\begin{array}{c}\text { Area } \\
1 \% \text { noise }\end{array}$ & $\begin{array}{c}\text { Area } \\
2 \% \text { noise }\end{array}$ & $\begin{array}{c}\text { Area } \\
3 \% \text { noise }\end{array}$ \\
\hline \multirow[t]{7}{*}{ Pediatric brain } & \multirow{4}{*}{$\mathrm{AP}$} & 1 & $339 \mathrm{~mm}^{2}$ & $820 \mathrm{~mm}^{2}$ & $2016 \mathrm{~mm}^{2}$ \\
\hline & & 2 & $4011 \mathrm{~mm}^{2}$ & $2060 \mathrm{~mm}^{2}$ & $1319 \mathrm{~mm}^{2}$ \\
\hline & & 3 & 5904 mm$^{2}$ & $3083 \mathrm{~mm}^{2}$ & $2122 \mathrm{~mm}^{2}$ \\
\hline & & 1 & $66 \mathrm{~mm}^{2}$ & $4 \mathrm{~mm}^{2}$ & $3 \mathrm{~mm}^{2}$ \\
\hline & \multirow[t]{2}{*}{ LR } & 2 & $2305 \mathrm{~mm}^{2}$ & $71 \mathrm{~mm}^{2}$ & $9 \mathrm{~mm}^{2}$ \\
\hline & & 3 & $5355 \mathrm{~mm}^{2}$ & $614 \mathrm{~mm}^{2}$ & $73 \mathrm{~mm}^{2}$ \\
\hline & \multirow{4}{*}{$\mathrm{AP}$} & 1 & $3 \mathrm{~mm}^{2}$ & - & - \\
\hline \multirow[t]{6}{*}{ Adult brain } & & 2 & $116 \mathrm{~mm}^{2}$ & $3 \mathrm{~mm}^{2}$ & - \\
\hline & & 3 & $1401 \mathrm{~mm}^{2}$ & $13 \mathrm{~mm}^{2}$ & - \\
\hline & & 1 & $336 \mathrm{~mm}^{2}$ & $63 \mathrm{~mm}^{2}$ & $22 \mathrm{~mm}^{2}$ \\
\hline & \multirow[t]{3}{*}{ LR } & 2 & $1683 \mathrm{~mm}^{2}$ & $370 \mathrm{~mm}^{2}$ & $134 \mathrm{~mm}^{2}$ \\
\hline & & 3 & $4535 \mathrm{~mm}^{2}$ & $1071 \mathrm{~mm}^{2}$ & $396 \mathrm{~mm}^{2}$ \\
\hline & & 1 & - & - & - \\
\hline \multirow[t]{3}{*}{ Prostate } & \multirow[t]{3}{*}{$\mathrm{AP}$} & 2 & $3.4 \%$ & - & - \\
\hline & & 3 & $44.2 \%$ & $1.1 \%$ & $0.1 \%$ \\
\hline & & 1 & $5.5 \%$ & $1.7 \%$ & $0.9 \%$ \\
\hline \multirow[t]{5}{*}{ Lung } & \multirow[t]{3}{*}{ AP1 } & 2 & $29.6 \%$ & $5.5 \%$ & $2.7 \%$ \\
\hline & & 3 & $78.8 \%$ & $14.5 \%$ & $5.5 \%$ \\
\hline & & 1 & $0.2 \%$ & - & - \\
\hline & \multirow[t]{2}{*}{ AP2 } & 2 & $18.2 \%$ & $0.2 \%$ & - \\
\hline & & 3 & $77.6 \%$ & $2.9 \%$ & - \\
\hline
\end{tabular}

Note: AP, anterior-posterior; LR, left-right; small fields, AP1 and AP2, are shown in Fig. 4. Areas greater than $2500 \mathrm{~mm}^{2}$ and percentages greater than $75 \%$ are shown in bold. 


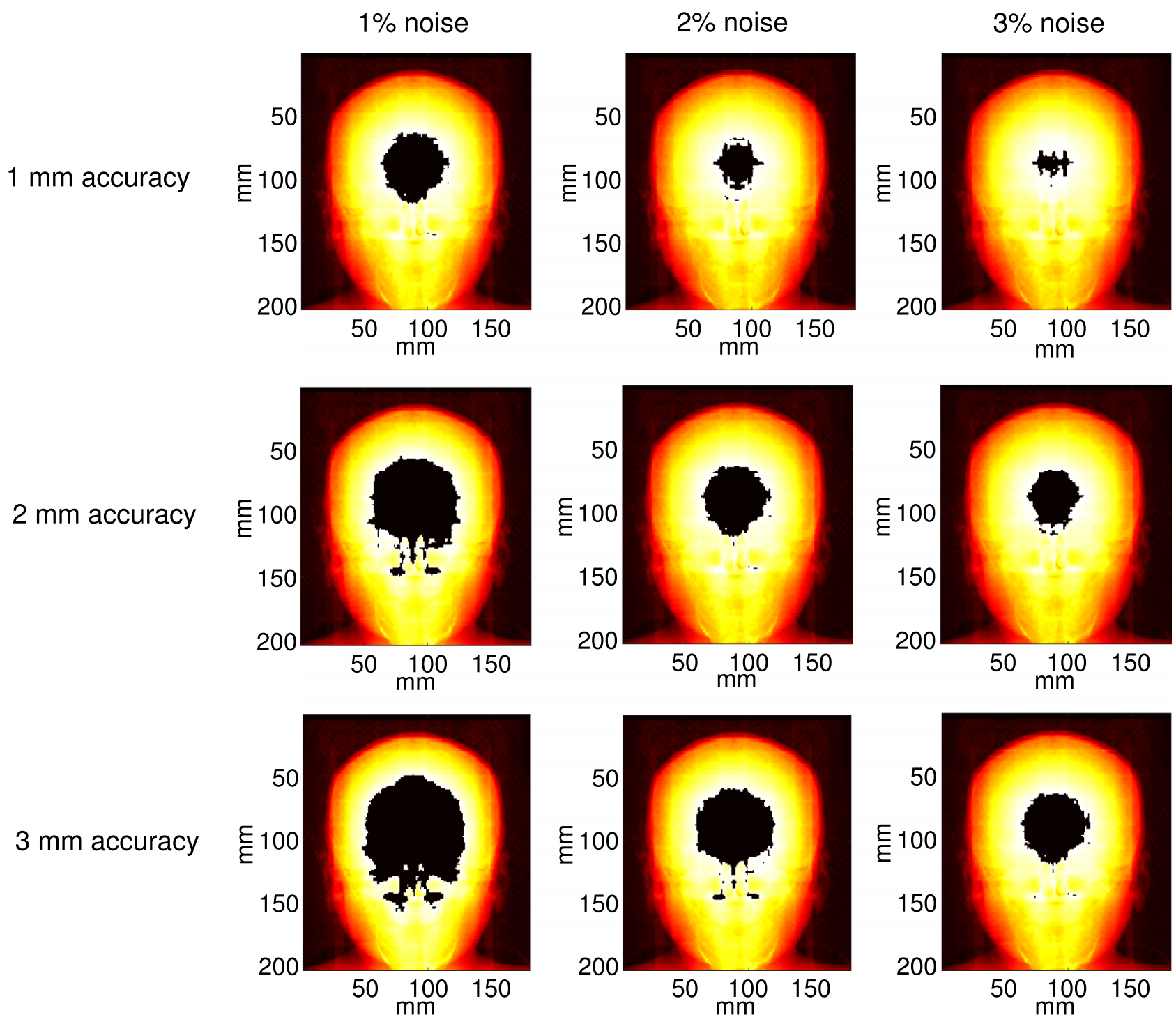

FIG. 9. Areas that can be imaged (shown by the dark region superimposed on brain) for an AP pediatric brain using dose ratio proton radiography.

given required WET accuracy, the user can then look up the proximal dynamic range for their energy pair (Fig. 8) and set up their measurement so that the object/region to be imaged has the correct total WET. Provided the object has a WET falling within this proximal dynamic range, as defined in Fig. 2, the accuracy remains within the required level.

It was found that the maximum proximal dynamic range for a given noise level was not obtained by the largest separation between the energy pair, rather there is an optimum value (typically $\sim 190 \mathrm{MeV}$ ). The reason for this can be explained with reference to Fig. 3. Although the proximal value of the dose ratio decreases with a greater separation in energies, at lower energies, there is less range straggling and so the Bragg peak is narrower, shortening the proximal dynamic range. The optimum value arises because, although it is beneficial to have a lower value for the dose ratio in the plateau region, some Bragg peak broadening is also desirable.

For comparison, the analysis was repeated on the distal side of the Bragg peak and distal dynamic ranges were calculated. Across all settings, the largest distal dynamic range was
$2.42 \mathrm{~cm}$ (lower energy of $220 \mathrm{MeV}, 5 \mathrm{~mm}$ required accuracy in the WET and $1 \%$ noise level). Comparatively, with the same required accuracy in the WET and a $1 \%$ noise level, a proximal dynamic range of $5.31 \mathrm{~cm}$ could be utilized. It can be seen from Fig. 8 that the use of the proximal side of the Bragg peak allows for the use of a larger dynamic range, but only for low noise levels $(1 \%-2 \%)$. When the noise levels become too high, the use of the distal side of the Bragg peak becomes more reliable and larger dynamic ranges can generally be utilized.

\section{B. Application to patient sites}

As mentioned in Sec. 2.A, most proton centers currently employ a margin of between $2.5 \%$ and $3.5 \%$ of the proton range to account for range uncertainty. ${ }^{7}$ Therefore, proton radiography would offer an improvement on current clinical practice if it could predict the proton range with an accuracy of less than $2.5 \%$, or $5 \mathrm{~mm}$ for a $20 \mathrm{~cm}$ range proton beam. Given the investment (both time and money) required to start using proton radiography routinely, the technique should offer 
(a)

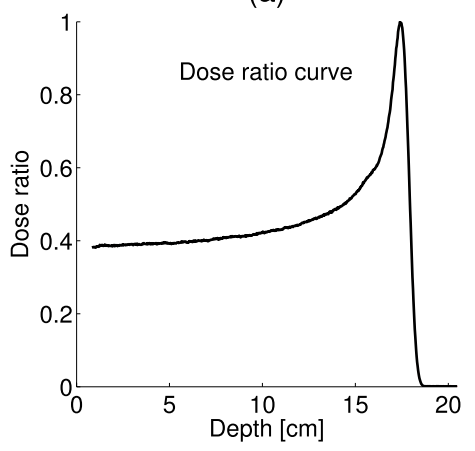

(d)

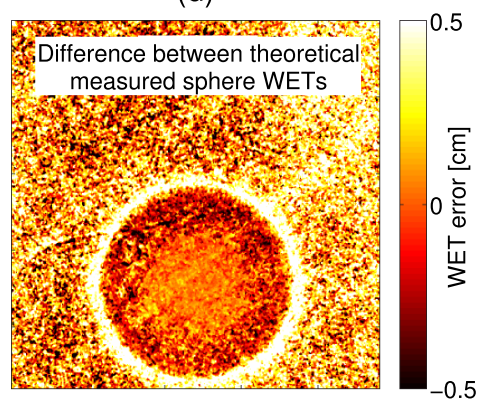

(b)

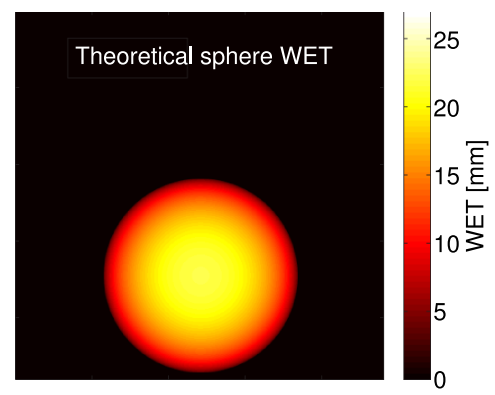

(c)

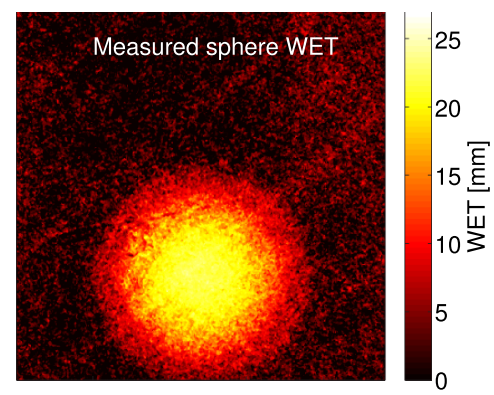

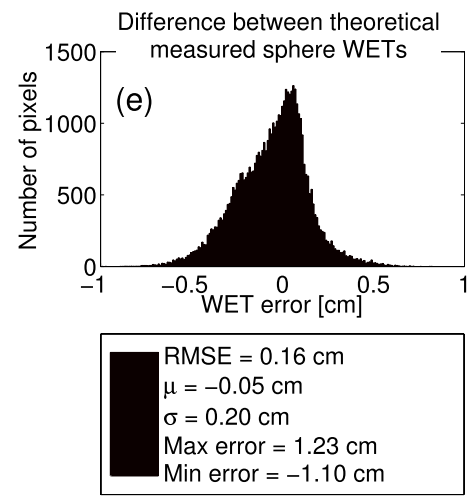

(f)

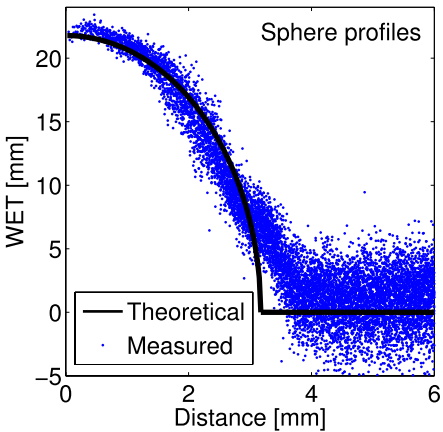

FIG. 10. (a) Measured dose ratio curve. (b) Theoretical and (c) measured WET maps for the sapphire sphere. (d) Difference (measured-theoretical) WET map. (e) Difference histogram within the sphere radius. (f) Theoretical and measured radial profiles of the $0.1 \mathrm{~mm}$ pixel WET maps. The $x$-axis is the distance from the sphere center.

a significant improvement on current clinical practice. We consider that a $1 \%$ accuracy, or $2 \mathrm{~mm}$ of a $20 \mathrm{~cm}$ range beam, would be a sufficiently high improvement to enable its introduction.

For such an accuracy, for a noise level in the dose ratio map of $1 \%$, dose ratio radiography on the proximal side of Bragg peak has a proximal dynamic range of up to $2.91 \mathrm{~cm}$ (dependent on the lower energy). This is quite a small range and it was found that the technique can only feasibly be applied to a specific set of patient sites, and within each site, only a specific region can be imaged. The following sites were tested for feasibility: brain (pediatric and adult, AP and LR); prostate

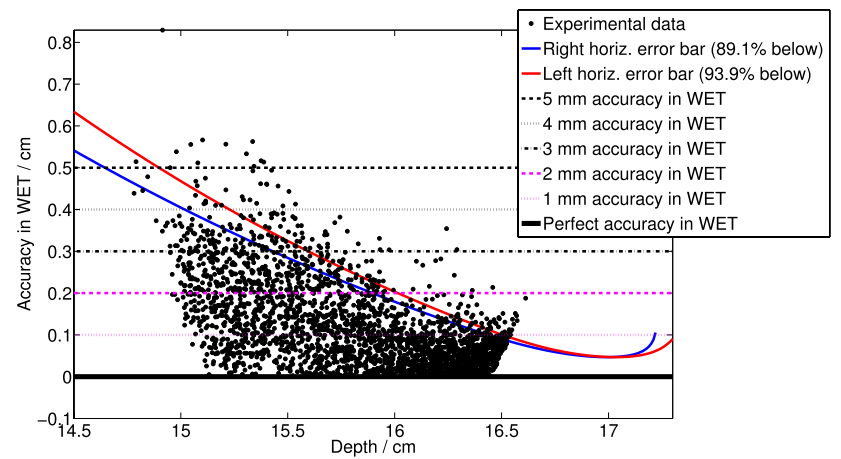

FIG. 11. Theoretical limits for a $2.35 \%(1.96 \sigma)$ noise in the dose ratio, for the energies used in measurements. The measured data of each $0.1 \mathrm{~mm}$ pixel are shown by the black dots. Given the measured data follow an approximately Gaussian distribution, we would expect $95 \%$ of the pixels to fall below the theoretical limits.
(AP only); and two small regions in the lung (AP only). The brain cases (in particular, the pediatric brain) demonstrated the best possible application of the technique. Provided the noise in the dose ratio map is no more than $1 \%$, equivalent square field sizes in the pediatric brain of $6.3 / 7.6 \mathrm{~cm}^{2}$ could be imaged under required accuracies of $2 / 3 \mathrm{~mm}$, respectively. It should be commented, however, that the shapes of these areas were not always connected (Fig. 9).

Although not explored in this work, it would be possible to increase the region that can be imaged by using additional energy pairs, although this has the drawback of increasing the patient dose. Another possibility is the use of a proton beam with a higher maximum energy. All of our analysis was based on using a fixed higher energy of $230 \mathrm{MeV}$, but some proton systems can generate energies up to $330 \mathrm{MeV}$. This would reduce the contribution of the higher beam to the dose ratio, which may allow for a larger dynamic range for imaging.

One disadvantage of the technique is the requirement of prior knowledge of the approximate WET of the patient site, so that the appropriate energy pair for imaging can be selected. The suggested approach for this is to convert the patient $\mathrm{x}$-ray CT dataset into a dataset of RSPs, and generate a DRR in units of WET that can then be used as an indication to select the best energy pair (as used in our work).

Another limitation of the technique is the effect of range straggling, which will increase in heterogeneous tissues. Such straggling effects will modify the depth dose distribution for a given energy, which will introduce additional uncertainties in the calculated WET. 


\section{C. Experimental demonstration in a single-detector CMOS APS}

Our experimental work demonstrated the use of the dose ratio method on the proximal side of the Bragg peak. We imaged a small sapphire sphere in a high energy proton beam line using a small $\left(13 \times 13 \mathrm{~mm}^{2}\right)$ CMOS APS sensor. Using two Bragg peaks, of 160.8 and $184.2 \mathrm{MeV}$, a dose ratio curve was formed and, after some image processing, a WET map was generated.

\section{C.1. Accuracy and suitability of CMOS APS}

After determining the precise sphere location, radial profiles of the measured data were compared to the theoretical WET radial profile, showing excellent agreement (RMSE $=1.6 \mathrm{~mm}$ within the theoretical sphere radius). This accuracy is not much worse than the previous works in which the distal edge of the Bragg peak was used (WET accuracy up to $0.9 \mathrm{~mm}){ }^{40}$ It can be seen in Figs. 10(d) and 10(f) that the measurements overestimate the sphere WET at the wider radii. This is likely to be caused by multiple Coulomb scattering, which has the effect of scattering the protons outward from the sphere center (where the WET is larger). This effect would have less of an impact if larger objects were imaged (and a larger CMOS sensor could be used to image them). Despite this, the CMOS APS demonstrated good suitability as a detector for dose ratio proton radiographic imaging, with a WET mean error of $0.5 \mathrm{~mm}$ (within the theoretical sphere radius).

As discussed above in Sec. 4.B, to be used clinically, the noise level in the dose ratio map generated needs to be not higher than $1 \%$. If it exceeds this level, the region that can be imaged (for the clinical sites assessed) is too small to prove useful. In this regard, the CMOS APS, with a $1.96 \sigma$ noise level of $2.35 \%$ (see Sec. 4.C.2 below), is probably too noisy.

Another problem of the CMOS APS used was the fact that it is not radiation hard. To be able to make reliable measurements, the sensitivity of the detector had to be characterized with a calibration image prior to each measurement. Although inconvenient, detector calibration would be required to account for other detector factors such as temperature and day-to-day fluctuations anyway. Provided the measurement is quick and automated, we do not consider this to be a big problem. Alternatively, radiation-hard detectors could be used or careful measurements of the sensitivity response of the detector with dose could be made.

\section{C.2. Validation of theoretical predictions}

The noise level in the dose ratio map was estimated from a calibration image in which a single solid water thickness was imaged to be $\sigma=1.2 \%$. Following the same procedure as the theoretical analysis of Sec. 2.A, theoretical error bars could be determined for the energy pair and for a noise level of $1.96 \sigma$. It was found that the measured error in the WET agreed with the theoretical predictions well, falling under the horizontal right/left errors bars $89.1 \% / 93.9 \%$ of the time (Fig. 11). These values are in good agreement with the prediction that a noise level of $1.96 \sigma$ should encompass $95 \%$ of the measured data points, given that the measured errors follow an approximately Gaussian shape. It can be concluded that the method of analysis to generate the theoretical predictions, in Secs. 2.A and 2.B, is appropriate.

\section{D. Study limitations}

One of the limitations of our theoretical application to patient sites is that the WET maps were generated using straight line DRRs, but it is well known that protons take longer paths due to the effect of multiple Coulomb scattering. This would have the effect of spreading the range of WETs in the patient, thus decreasing the region of the patient we could image. A potential solution to this, as suggested in Sec. 4.B, would be to use multiple energy pairs, with each pair designed to image different regions.

Our theoretical analysis was verified using a CMOS APS. However, because of the small detector size, $13 \times 13 \mathrm{~mm}$ square, we were restricted to imaging objects with a small maximum WET (a sapphire sphere with a maximum WET of $21.8 \mathrm{~mm}$ was imaged). It is expected that the verification would still hold at high WET, but this requires confirmation, particularly as the WETs used in the theoretical analysis were much higher (maximum $242 \mathrm{~mm}$ ).

\section{E. Outlook of the dose ratio technique}

Based on this theoretical analysis, the potential use of this technique appears quite limited. Only in the imaging of sites with a small maximum WET, namely, pediatrics, it is possible to image reasonably sized fields (greater than an equivalent $5 \times 5 \mathrm{~cm}^{2}$ square). This also requires the accuracy in the WET to be no stricter than $2 \mathrm{~mm}$, and the user to have a detector with a noise level of no higher than $1 \%$. In addition to the limited dynamic range of this technique, it requires the measurement of dose from two pristine Bragg peaks, and consequently twice the amount of dose with respect to any radiographic techniques that use only one. If, as has been suggested previously, the dynamic range was to be increased using additional energy pairs, the dose would be increased further.

Despite these limitations, the technique does have some advantages. First, it only requires the use of a single detector, which, as discussed in the Introduction, can be more conveniently introduced into the clinical environment than classical proton radiographic devices. Additionally, it provides a potential solution for PBS, which appears to increasingly become the standard mode of delivery for proton therapy. Dose was not explored in this work, but given that relative images are required, it may be possible to deliver a very small dose for each energy (provided the signal-to-noise ratio is not too low).

\section{SUMMARY}

We have systematically assessed the dose ratio method using a set of theoretical pristine Bragg peaks. We generated lookup graphs that have listed the range that one can use on the proximal side of the Bragg peak, dependent on the energy pair, 
noise level in the dose ratio image, and the required accuracy in the WET. We showed that, provided the noise level is less than $2 \%$, the proximal side of the Bragg peak has a larger dynamic range (maximum $5.31 \mathrm{~cm}$ ) than when using the distal side of the Bragg peak (maximum $2.42 \mathrm{~cm}$ ).

We have shown that use of the dose ratio on the proximal side of the Bragg peak has some potential as a proton radiographic method, although the choice of patient sites is critical. For an example pediatric brain, it is possible to use the technique to image a region with a square field equivalent size of $7.6 \mathrm{~cm}^{2}$, for a $3 \mathrm{~mm}$ accuracy in the WET and $1 \%$ noise level in the dose ratio image.

We validated these theoretical predictions with experimental measurements using a CMOS APS, which was found to have a noise of $\sigma=1.2 \%$. The CMOS APS proved to be a sufficiently accurate detector for this technique, with a RMSE of $1.6 \mathrm{~mm}$ WET in the imaging of a small sapphire sphere.

\section{ACKNOWLEDGMENTS}

The authors would like to acknowledge the support of the operators at Massachusetts General Hospital. This project was funded equally by the Engineering and Physical Sciences Research Council (UK) and Ion Beam Applications (LouvainLa-Neuve, Belgium).

a) Author to whom correspondence should be addressed. Electronic mail: paul.doolan.09@ucl.ac.uk

${ }^{1}$ A. Cormack, "Representation of a function by its line integrals, with some radiological applications," J. Appl. Phys. 34(9), 2722-2727 (1963).

${ }^{2}$ R. Schulte et al., "Density resolution of proton computed tomography," Med. Phys. 32(4), 1035-1046 (2005)

${ }^{3} \mathrm{~J}$. Seco and N. Depauw, "Proof of principle study of the use of a CMOS active pixel sensor for proton radiography,” Med. Phys. 38(2), 622-623 (2011).

${ }^{4} \mathrm{U}$. Schneider et al., "First proton radiography of an animal patient," Med. Phys. 31(5), 1046-1051 (2004).

${ }^{5}$ R. F. Hurley et al., "Water-equivalent path length calibration of a prototype proton CT scanner," Med. Phys. 39(5), 2438-2446 (2012).

${ }^{6} \mathrm{~B}$. Schaffner and E. Pedroni, "The precision of proton range calculations in proton radiotherapy treatment planning: Experimental verification of the relation between CT-HU and proton stopping power," Phys. Med. Biol. 43, 1579-1592 (1998).

${ }^{7} \mathrm{H}$. Paganetti, "Range uncertainties in proton therapy and the role of Monte Carlo simulations,” Phys. Med. Biol. 57(11), R99-R117 (2012).

${ }^{8}$ U. Schneider, P. Pemler, J. Besserer, E. Pedroni, A. Lomax, and B. KaserHotz, "Patient specific optimization of the relation between CT-Hounsfield units and proton stopping power with proton radiography," Med. Phys. 32(1), 195-199 (2005).

${ }^{9}$ N. Depauw, M. F. Dias, A. Rosenfeld, and J. C. Seco, "Ion radiography as a tool for patient set-up \& image guided particle therapy: A Monte Carlo study," Technol. Cancer Res. Treat. 13, 69-76 (2014).

${ }^{10} \mathrm{M}$. Testa et al., "Proton radiography and proton computed tomography based on time-resolved dose measurements," Phys. Med. Biol. 58(22), 8215-8233 (2013).

${ }^{11}$ U. Schneider, J. Besserer, and M. Hartmann, “Technical note: Spatial resolution of proton tomography: Impact of air gap between patient and detector," Med. Phys. 39(2), 798-800 (2012).

${ }^{12} \mathrm{P}$. Pemler et al., "A detector system for proton radiography on the gantry of the Paul-Scherrer-Institute," Nucl. Instrum. Methods Phys. Res., Sect. A 432(2-3), 483-495 (1999).

${ }^{13}$ H. Shinoda, T. Kanai, and T. Kohno, "Application of heavy-ion CT," Phys. Med. Biol. 51(16), 4073-4081 (2006).

${ }^{14} \mathrm{R}$. Schulte et al., "Conceptual design of a proton computed tomography system for applications in proton radiation therapy,' IEEE Trans. Nucl. Sci. 51(3), 866-872 (2004).
${ }^{15}$ C. Talamonti et al., "Proton radiography for clinical applications," Nucl. Instrum. Methods Phys. Res., Sect. A 612(3), 571-575 (2010).

${ }^{16}$ V. Sipala et al., "PRIMA: An apparatus for medical application," Nucl. Instrum. Methods Phys. Res., Sect. A 658, 73-77 (2011).

${ }^{17}$ G. Poludniowski et al., "Proton-counting radiography for proton therapy: A proof of principle using CMOS APS technology," Phys. Med. Biol. 59(11), 2569-2581 (2014).

${ }^{18}$ P. S. Friedman et al., "Plasma panel sensors for particle and beam detection," IEEE Nuclear Science Symposium Medical Imaging Conference Record, Anaheim, CA (IEEE, 2012), pp. 1775-1780.

${ }^{19}$ R. Ball et al., "Development of a plasma panel radiation detector," Nucl. Instrum. Methods Phys. Res., Sect. A 764, 122-132 (2014).

${ }^{20} \mathrm{~S}$. Braccini et al., "First results on proton radiography with nuclear emulsion detectors," J. Instrum. 5(09), 1-14 (2010).

${ }^{21}$ D. Watts et al., "A proton range telescope for quality assurance in hadrontherapy," in IEEE Nuclear Science Symposium Conference Record, Orlando, FL (IEEE, 2009), pp. 4163-4166.

${ }^{22} \mathrm{R}$. Hollebeek et al., "A new technology for fast two-dimensional detection of proton therapy beams," Phys. Res. Int. 2012(i), 1-11 (2012).

${ }^{23}$ M. Akisada, J. Ohashi, and K. Kondo, "Conceptual design of proton computed tomography with magnetic spectrometer," Jpn. J. Appl. Phys. 22(4), 752-758 (1983).

${ }^{24} \mathrm{U}$. Schneider and E. Pedroni, "Proton radiography as a tool for quality control in proton therapy," Med. Phys. 22(4), 353-363 (1995).

${ }^{25}$ J. Heimann, "Developing an FPGA-based readout for the pCT detector system" (University of California Berkeley, CA, 2005).

${ }^{26} \mathrm{M}$. Petterson et al., "Proton radiography studies for proton CT," IEEE Nuclear Science Symposium Conference Record, San Diego, CA (IEEE, 2006), pp. 2276-2280.

${ }^{27}$ E. Vanzi et al., "The PRIMA collaboration: Preliminary results in FBP reconstruction of pCT data," Nucl. Instrum. Methods Phys. Res., Sect. A 730, 184-190 (2013).

${ }^{28}$ V. A. Bashkirov, R. W. Schulte, S. N. Penfold, S. Member, and A. B. Rosenfeld, "Proton computed tomography: Update on current Status," IEEE Nuclear Science Symposium Conference Record HT6-1, Honolulu, HI (IEEE, 2007), pp. 4685-4688.

${ }^{29}$ I. Rinaldi et al., "Experimental characterization of a prototype detector system for carbon ion radiography and tomography," Phys. Med. Biol. 58(3), 413-427 (2013).

${ }^{30}$ I. Rinaldi, S. Brons, O. Jäkel, B. Voss, and K. Parodi, "Experimental investigations on carbon ion scanning radiography using a range telescope," Phys. Med. Biol. 59(12), 3041-3057 (2014).

${ }^{31}$ H. Ryu, E. Song, J. Lee, and J. Kim, "Density and spatial resolutions of proton radiography using a range modulation technique," Phys. Med. Biol. 53(19), 5461-5468 (2008).

${ }^{32} \mathrm{H}$. Muraishi et al., "Evaluation of spatial resolution for heavy ion CT system based on the measurement of residual range distribution with HIMAC," IEEE Trans. Nucl. Sci. 56(5), 2714-2721 (2009).

${ }^{33}$ E. Bentefour, D. Samuel, M. Testa, and H.-M. Lu, "Methods and device for dose based proton radiography," Med. Phys. 40(6), 308 (2013).

${ }^{34}$ J. Telsemeyer, O. Jäkel, and M. Martišíková, "Quantitative carbon ion beam radiography and tomography with a flat-panel detector," Phys. Med. Biol. 57(23), 7957-7971 (2012).

${ }^{35}$ E. Gelover-Reyes, F. Jimenez-Sprang, I. Rosenberg, D. D'Souza, and G. Royle, "Proton radiography with silicon active sensors," in ESTRO Proceedings (ESTRO, London, 2011), p. S531.

${ }^{36} \mathrm{H}$.-M. Lu, "A point dose method for in vivo range verification in proton therapy," Phys. Med. Biol. 53(23), N415-N422 (2008).

${ }^{37}$ H.-M. Lu, G. Mann, and E. Cascio, "Investigation of an implantable dosimeter for single-point water equivalent path length verification in proton therapy," Med. Phys. 37(11), 5858 (2010).

${ }^{38}$ H.-M. Lu, "A potential method for in vivo range verification in proton therapy treatment," Phys. Med. Biol. 53(5), 1413-1424 (2008).

${ }^{39}$ B. Gottschalk, S. Tang, E. H. Bentefour, E. Cascio, D. Prieels, and H.-M. $\mathrm{Lu}$, "Water equivalent path length measurement in proton radiotherapy using time resolved diode dosimetry," Med. Phys. 38(4), 2282-2288 (2011).

${ }^{40}$ E. H. Bentefour, M. Testa, and H.-M. Lu, "In-vivo WEPL verification based on point dose measurements in proton treatment by beam scanning," in PTCOG (PTCOG, 2012), Vol. 51, p. 3855.

${ }^{41} \mathrm{~T}$. Bortfeld, "An analytical approximation of the Bragg curve for therapeutic proton beams," Med. Phys. 24(12), 2024-2033 (1997).

${ }^{42} \mathrm{~S}$. Bohndiek et al., "Characterization studies of two novel active pixel sensors," Opt. Eng. 46(12), 124003 (2007). 
${ }^{43}$ S. A. Basun, R. S. Meltzer, and G. F. Imbusch, "Exchange-coupled chromium ion pairs in ruby revisited," J. Lumin. 125(1-2), 31-39 (2007).

${ }^{44} \mathrm{~S}$. Ganschow, D. Klimm, and R. Bertram, "On the effect of oxygen partial pressure on the chromium distribution coefficient in melt-grown ruby crystals," J. Cryst. Growth 325(1), 81-84 (2011).

${ }^{45}$ J. Boone and A. Chavez, "Comparison of x-ray cross sections for diagnostic and therapeutic medical physics," Med. Phys. 23(12), 1997-2005 (1996).

${ }^{46}$ J. Berger and M. Zucker, ESTAR, PSTAR, and ASTAR: Computer Programs for Calculating Stopping-Power and Range Tables for Electrons, Protons, and Helium Ions (version 1.2.2), National Institute of Standards and Technology, 2004, available: http://physics.nist.gov/Star.

${ }^{47}$ N. Hünemohr, B. Krauss, C. Tremmel, B. Ackermann, O. Jäkel, and S. Greilich, "Experimental verification of ion stopping power prediction from dual energy CT data in tissue surrogates," Phys. Med. Biol. 59(1), 83-96 (2014).
${ }^{48}$ M. Esposito, T. Anaxagoras, and O. Diaz, "Radiation hardness of a large area CMOS Active Pixel Sensor for bio-medical applications," IEEE Nuclear Science Symposium Conference Record, Anaheim, CA (IEEE, 2012), Vol. N14, Iss. 183, pp. 1300-1304

${ }^{49}$ S. Meroli, D. Passeri, L. Servoli, and A. Angelucci, "Analysis of the performance of CMOS APS imagers after proton damage," J. Instrum. 8(02), 1-5 (2013).

${ }^{50} \mathrm{M}$. Esposito et al., "Ionizing and non ionizing radiation damage in a large area CMOS active pixel sensor for medical applications," IEEE Trans. Nucl. Sci. 1-8 (2014), available at http://eprints.lincoln.ac.uk/14148/.

${ }^{51}$ G. D. Stewart, Silicon Pixel Detectors for Synchrotron Applications (University of Glasgow, Scotland, 2013).

${ }^{52}$ A. C. Konstantinidis, A. Olivo, P. R. T. Munro, S. E. Bohndiek, and R. D. Speller, "Optical characterisation of a CMOS active pixel sensor using periodic noise reduction techniques," Nucl. Instrum. Methods Phys. Res., Sect. A 620, 549-556 (2010). 